\title{
Los cambios del estado físico y el índice de masa corporal influyen en la mortalidad total y cardiovascular en hombres
}

Changes in fitness and body mass index influence total and cardiovascular mortality in men

\section{Objetivos}

Analizar el efecto de los cambios del estado físico cardiopulmonar (EFC; traducido del inglés fitness, y entendido como grado de entrenamiento físico) y del índice de masa corporal (IMC) sobre la mortalidad total y cardiovascular, en forma independiente y/o combinada.

\section{Diseño}

Estudio prospectivo observacional de una cohorte de 14.345 hombres (integrantes del Aerobics Center Longitudinal Study), que fueron evaluados al menos en dos oportunidades en un intervalo de 28 años. El seguimiento promedio posterior a la última evaluación fue de 11,4 años.

\section{Lugar y Pacientes}

Los participantes fueron referidos o concurrieron espontáneamente a la Clínica Cooper de Dallas, entre 1974 y 2002. Eran hombres blancos, no hispanos, mayores de 20 años, con estudios universitarios y de clase media a alta. La media de edad fue de 43,7 \pm 9,1 años, se hallaban en buen estado físico (METs medios basales 11,9) y con un ligero sobrepeso: IMC basal: $26,0 \mathrm{Kg} / \mathrm{m} 2$.

\section{Evaluación de factores pronósticos}

En el periodo de evaluación se determinó, entre otros, el IMC a través de la toma de medidas antropométricas, y el EFC a través de una prueba ergométrica (cuyo resultado se expresó en METs). En el caso de que el participante hubiese realizado más de dos evaluaciones, se contabilizó el cambio ocurrido entre la primera y la última medición. En la fase de seguimiento se determino la mortalidad total y de causa cardiovascular.

\section{Resultados principales}

Los cambios registrados en el EFC e IMC fueron divididos en tres categorías homogéneas: pérdida, mantenimiento e incre- mento. En la tabla 1 pueden verse resumidos los principales resultados, tomando la categoría pérdida como punto de comparación.

Tabla 1: Mortalidad general y específica según cambios en el estado físico cardiovascular e índice de masa corporal.

\begin{tabular}{|c|c|c|c|c|}
\hline \multirow{2}{*}{$\begin{array}{c}\text { Categoria de } \\
\text { cambio }\end{array}$} & \multicolumn{2}{|c|}{$\begin{array}{l}\text { Mortalidad por todas las } \\
\text { causas } \mathrm{HR}^{\star \star}(\mathrm{IC95} \%)\end{array}$} & \multicolumn{2}{|c|}{$\begin{array}{l}\text { Mortalidad de origen } \\
\text { cardiovascular HR** (IC95\%) }\end{array}$} \\
\hline & $E F C_{ \pm}$ & IMC & $E F G \pm$ & IMC \\
\hline Perdida & 1,00 & 1,00 & 1,00 & 1,00 \\
\hline Mantenimiento & $\begin{array}{c}0,70 \\
(0,59 \text { a 0,82) }\end{array}$ & $\begin{array}{c}1,14 \\
(0,96 \text { a } 1,35) \\
\end{array}$ & $\begin{array}{c}0,72 \\
(0,54 \text { a } 0,97)\end{array}$ & $\begin{array}{c}1,04 \\
(0,76 \text { a } 1,42)\end{array}$ \\
\hline Incremento & $\begin{array}{c}0,60 \\
(0,50 \mathrm{a} 0,71)\end{array}$ & $\begin{array}{c}1,15 \\
(0,98 \text { a } 1,36) \\
\end{array}$ & $\begin{array}{c}0,56 \\
(0,41 \text { a } 0,76)\end{array}$ & $\begin{array}{c}1,39 \\
(1,05 \text { a } 1,84)\end{array}$ \\
\hline $\begin{array}{l}\mathrm{x1} \text { MET } \\
\text { incremento }\end{array}$ & $\begin{array}{c}0,84 \\
(0,80 \mathrm{a} 0,88)\end{array}$ & - & $\begin{array}{c}0,80 \\
(0,74 \text { a } 0,88)\end{array}$ & - \\
\hline $\begin{array}{l}\text { x1 IMC } \\
\text { incremento }\end{array}$ & - & $\begin{array}{c}1,06 \\
(1,01 \mathrm{a} 1,10) \\
\end{array}$ & - & $\begin{array}{c}1,08 \\
(1,01 \text { a } 1,17)\end{array}$ \\
\hline
\end{tabular}

**Ajustado por: edad; año de evaluación; antecedentes familiares de enfermedad cardiovascular precoz; IMC y METs basales; tabaquismo, consumo de alcohol y actividad física; alteraciones electrocardiográficas, hipertensión, diabetes, e hipercolesterolemia y número de consultas médicas entre en la primera y última evaluaciones. \pm Estos resultados se mantuvieron significativos aun al incorporar al ajuste la variable cambio en IMC.

\section{Conclusión}

Mantener o mejorar el EFC en hombres se asocia con el menor riesgo de mortalidad general y por causa cardiovascular. La prevención de la pérdida del EFC asociado a la edad aporta a aumentar la longevidad, independientemente de los cambios en el IMC.

Fuente de financiamiento: Instituto Nacional de Salud de EE.UU y The Coca-Cola Company.

\section{Comentario}

La detección del EFC como factor protector o su espejo contrario, el sedentarismo, como factor de riesgo de mortalidad total y cardiovascular independiente, llevó un largo pero lógico recorrido. Primero fueron estudios epidemiológicos retrospectivos, después de cohorte con una sola medición del EFC, para finalmente llegar a los actuales donde no solo es ponderado el EFC sino la presencia de sobrepeso y obesidad y sus variaciones en el tiempo. El articulo comentado no es el primero de esta clase, pero si el de mayor población y seguimiento que confirma los hallazgos de sus predecesores.

\section{Conclusiones del comentador}

En nuestra práctica cotidiana debemos estimular la práctica regular de actividad física y/o deportes, en todos nuestros pacientes. Incluso en aquellos con sobrepeso y obesidad un adecuado EFC disminuye el riesgo de enfermedades cardiovasculares.

Diego Iglesias [ Servicio de Cardiología. Hospital Italiano de Buenos Aires. diego.iglesias@hospitalitaliano.org.ar ]

Iglesias D. Los cambios del estado físico y el índice de masa corporal influyen en la mortalidad total y cardiovascular en hombres. Evid Act Pract Amb Ene-Mar 2012;15(1):14. Comentado de: Lee DC, Sui X, Artero EG, y col. Long-term effects of changes in cardiorespiratory fitness and body mass index on all-cause and cardiovascular disease mortality in men: the Aerobics Center Longitudinal Study. Circulation.2011;124:2483-2490. PMID: 22144631. 